ESAIM: COCV 22 (2016) 1163-1183

DOI: $10.1051 / \mathrm{cocv} / 2016035$
ESAIM: Control, Optimisation and Calculus of Variations

www.esaim-cocv.org

\title{
EXACT BOUNDARY SYNCHRONIZATION FOR A COUPLED SYSTEM OF 1-D QUASILINEAR WAVE EQUATIONS *,**,***
}

\author{
Long $\mathrm{Hu}^{1,2,3}$, TATsien $\mathrm{Li}^{4}$ AND Peng Qu ${ }^{2}$
}

\begin{abstract}
Based on the theory of semi-global classical solutions for quasilinear hyperbolic systems, under suitable hypotheses, an iteration procedure given by a unified constructive method is presented to establish the exact boundary synchronization for a coupled system of 1-D quasilinear wave equations with boundary conditions of various types.
\end{abstract}

Mathematics Subject Classification. 93B05, 35L04.

Received June 6, 2016. Accepted June 7, 2016.

\section{INTRODUCTION AND MAIN RESULT}

Synchronization is a widespread natural phenomenon. How to describe the phenomenon of synchronization for a coupled system has been widely studied by biologists, engineers and sociologists (see $[4,18]$ ). The previous research activities, however, only focused on the systems governed by ordinary differential equations or complex networks (see $[1,17,21]$ ). Recently, several kinds of exact synchronizations are introduced by Li et al. (see [3,14]) for a coupled system of 1-D linear wave equations with boundary conditions of Dirichlet type, Neumann type, coupled third type and coupled dissipative type in the framework of $C^{2}$ solutions, and it is shown that these synchronizations can be realized by means of a reduced number of boundary controls. For the multi-dimensional case, by indirectly using the HUM method (see $[15,16]$ ), a similar result for a coupled system of linear wave equations with Dirichlet boundary controls can be also established in the framework of weak solutions (see [11]). In this paper, based on the theory of semi-global classical solutions for quasilinear hyperbolic systems, under suitable hypotheses, we will present an iteration procedure given by a unified constructive method to establish

Keywords and phrases. Exact boundary synchronization, coupled system of quasilinear wave equations.

* Supported by the China Scholarship Council for Ph.D. study at UPMC (No. 201306100081) and the ERC advanced grant 266907 (CPDENL) of the 7th Research Framework Programme (FP7).

** Supported by the National Basic Research Program of China (No. 2013CB834100) and the National Natural Science Foundation of China (No. 11121101).

*** Supported by Shanghai Key Laboratory for Contemporary Applied Mathematics, Fudan University, the initiative funding for new researchers, Fudan University and Yang Fan Foundation of Shanghai on Science and Technology (No. 15YF1401100).

1 School of Mathematics, Shandong University, Jinan, Shandong 250100, P.R. China.

2 School of Mathematical Sciences, Fudan University, Shanghai 200433, P.R. China. pqu@fudan.edu.cn

3 Sorbonne Universités, UPMC Univ Paris 06, UMR 7598, Laboratoire Jacques-Louis Lions, 75005 Paris, France. hul10@fudan.edu.cn; hu@ann.jussieu.fr

4 School of Mathematical Sciences, Fudan University; Shanghai Key Laboratory for Contemporary Applied Mathematics; Nonlinear Mathematical Modeling and Methods Laboratory, Shanghai 200433, P.R. China. dqli@fudan.edu.cn 
the local exact boundary synchronization for a coupled system of 1-D quasilinear wave equations with afore mentioned boundary conditions.

Precisely speaking, we consider the following coupled system of quasilinear wave equations with a common propagation speed

$$
\frac{\partial^{2} U}{\partial t^{2}}-c^{2}\left(U, U_{t}, U_{x}\right) \frac{\partial^{2} U}{\partial x^{2}}+A\left(U, U_{t}, U_{x}\right) U=0
$$

on the domain $R(T)=\{(t, x) \mid 0 \leq t \leq T, 0 \leq x \leq L\}$, where $L$ is the length of the space interval, $T>0$ is a suitable number, $U=\left(u_{1}, \ldots, u_{N}\right)^{T}$ is an unknown vector function of $(t, x), A\left(U, U_{t}, U_{x}\right)=\left(a_{i j}\left(U, U_{t}, U_{x}\right)\right)$ is an $N \times N$ coupling matrix, whose elements are $C^{1}$ functions with respect to their arguments, $c\left(U, U_{t}, U_{x}\right)$, the common propagation speed for the coupled system, is also a $C^{1}$ function with respect to its arguments, and

$$
c(0,0,0)>0 .
$$

(1.1) can be written as:

$$
\frac{\partial^{2} u_{i}}{\partial t^{2}}-c^{2}\left(U, U_{t}, U_{x}\right) \frac{\partial^{2} u_{i}}{\partial x^{2}}+\sum_{j=1}^{N} a_{i j}\left(U, U_{t}, U_{x}\right) u_{j}=0 \quad(i=1, \ldots, N) .
$$

For fixing the idea, we study only the case of one-sided controls, the case of two-sided controls can be similarly treated (see Rem. 5.1). At the end $x=0$, we prescribe any one of the following boundary conditions:

$$
\begin{aligned}
& x=0: U=H(t) \text { (Dirichlet type), } \\
& x=0: U_{x}=H(t) \text { (Neumann type), } \\
& x=0: U_{x}-B(U) U=H(t) \text { (Coupled third type), }
\end{aligned}
$$

where $B(U)=\left(b_{i j}(U)\right)$ is an $N \times N$ boundary coupling matrix with $C^{1}$ elements, and

$$
H(t)=\left(h_{1}(t), \ldots, h_{N}(t)\right)^{T}
$$

are $C^{2}$ (case (1.4a)) or $C^{1}$ (cases (1.4b) and (1.4c)) functions of $t$, the components of which will be totally or partially taken as boundary controls.

At the end $x=L$, since no boundary controls are concerned, we prescribe any one of the following homogeneous boundary conditions:

$$
\begin{aligned}
& x=L: U=0 \text { (Dirichlet type) } \\
& x=L: U_{x}=0 \text { (Neumann type), } \\
& x=L: U_{x}+\bar{B}(U) U=0 \text { (Coupled third type), }
\end{aligned}
$$

where $\bar{B}(U)=\left(\bar{b}_{i j}(U)\right)$ is an $N \times N$ boundary coupling matrix with $C^{1}$ elements.

The initial condition is given by:

$$
t=0:\left(U, U_{t}\right)=(\Phi(x), \Psi(x)), 0 \leq x \leq L,
$$

in which $\Phi=\left(\varphi_{1}, \ldots, \varphi_{N}\right)^{T}$ and $\Psi=\left(\psi_{1}, \ldots, \psi_{N}\right)^{T}$ are $C^{2}$ and $C^{1}$ vector functions of $x$ on $[0, L]$, respectively, such that the conditions of $C^{2}$ compatibility at the points $(t, x)=(0,0)$ and $(0, L)$ are satisfied, respectively. Moreover, we assume that

$$
\|(\Phi, \Psi)\|_{\left(C^{2}[0, L]\right)^{N} \times\left(C^{1}[0, L]\right)^{N}} \leq \varepsilon
$$

where $\varepsilon>0$ is suitably small. 
Definition 1.1. The coupled system (1.1), (1.4) and (1.6) is said to possess the exact boundary synchronization if there exist two positive constants $T_{0}$ and $T$ with $T>T_{0}$, such that for any given initial state $(\Phi(x), \Psi(x))$ with small norm $\|(\Phi, \Psi)\|_{\left(C^{2}[0, L]\right)^{N} \times\left(C^{1}[0, L]\right)^{N}}$ and satisfying the conditions of $C^{2}$ compatibility at the point $(t, x)=(0, L)$, we can find some boundary controls with support on $\left[0, T_{0}\right]$ in $H(t)$, such that the corresponding mixed initial-boundary value problem (1.1), (1.4), (1.6) and (1.7) admits a unique $C^{2}$ solution $U(t, x)=\left(u_{1}(t, x), \ldots, u_{N}(t, x)\right)^{T}$ on the domain

$$
R(T)=\{(t, x) \mid 0 \leq t \leq T, 0 \leq x \leq L\}
$$

and as $T_{0} \leq t \leq T$ we have

$$
u_{1}(t, x) \equiv \ldots \equiv u_{N}(t, x) \stackrel{\text { def. }}{=} \widetilde{\widetilde{u}}(t, x), \quad 0 \leq x \leq L .
$$

$\widetilde{\widetilde{u}}=\widetilde{\widetilde{u}}(t, x)$, being a priori unknown, is called the corresponding synchronizable state.

Remark 1.2. The exact boundary null controllability of the coupled system (1.1), (1.4) and (1.6), which can be obtained by $N$ boundary controls $(c f .[2,10])$, provides a trivial example of the exact boundary synchronization. In this paper, however, our attention is focused on the exact boundary synchronization realized by means of only $(N-1)$ boundary controls.

For the exact boundary synchronization for a coupled system of 1-D linear wave equations, it is shown (cf. [14]) that if the system is not exactly boundary null controllable, then there is a necessary condition that the sum of the elements in every row of the coupling matrices $A=\left(a_{i j}\right), B=\left(b_{i j}\right)$ and $\bar{B}=\left(\bar{b}_{i j}\right)$ should be independent of $i=1, \ldots, N$. In this paper, for the corresponding quasilinear case, we impose the similar assumption that

$$
\begin{aligned}
& \sum_{j=1}^{N} a_{i j}\left(U, U_{t}, U_{x}\right) \stackrel{\text { def. }}{=} \widetilde{\widetilde{a}}\left(U, U_{t}, U_{x}\right), \\
& \sum_{j=1}^{N} b_{i j}(U) \stackrel{\text { def. }}{=} \widetilde{\widetilde{b}}(U), \\
& \sum_{j=1}^{N} \bar{b}_{i j}(U) \stackrel{\text { def. }}{=} \widetilde{\widetilde{b}}(U),
\end{aligned}
$$

where $\widetilde{\widetilde{a}}, \widetilde{\widetilde{b}}$ and $\widetilde{\bar{b}}$ are all independent of $i=1, \ldots, N$.

Obviously, $U=0$ is an equilibrium of system (1.1) with boundary conditions (1.4) (in which $H \equiv 0$ ) and (1.6). Based on the theory of semi-global $C^{2}$ solutions, using a unified constructive method for one-sided exact boundary controllability (see $[2,6,8,9]$ ), by a suitable iteration procedure as well as certain estimates for coupled system of wave equations, we will establish the local exact boundary synchronization around $U=0$. The main result in this paper is:

Theorem 1.3. Suppose that $c, a_{i j}, b_{i j}$ and $\bar{b}_{i j}$ are all $C^{1}$ functions with respect to their arguments on the domain under consideration. Suppose furthermore that (1.2) and (1.11)-(1.13) hold. Let

$$
T>T_{0}>2 \frac{L}{c(0,0,0)} .
$$

For any given initial state $(\Phi(x), \Psi(x)) \in\left(C^{2}[0, L]\right)^{N} \times\left(C^{1}[0, L]\right)^{N}$, satisfying simultaneously (1.8), the conditions of $C^{2}$ compatibility at the point $(t, x)=(0, L)$, as well as the conditions of $C^{2}$ compatibility at the 
point $(t, x)=(0,0)$ for the boundary conditions without controls in $(1.4)$, there exist $(N-1)$ boundary controls with support on $\left[0, T_{0}\right]$ in $H(t)$ (for example, we take $h_{2}(t), \ldots, h_{N}(t)$ as controls with $h_{1}(t) \equiv 0$ ) with small $\left(C^{2}[0, T]\right)^{N}$ norm (case (1.4a)) or $\left(C^{1}[0, T]\right)^{N}$ norm (cases $\left.(1.4 \mathrm{~b})-(1.4 \mathrm{c})\right)$, such that the mixed initialboundary value problem (1.1), (1.4) and (1.6)-(1.7) admits a unique $C^{2}$ solution $U=U(t, x)$ on the domain $R(T)=\{(t, x) \mid 0 \leq t \leq T, 0 \leq x \leq L\}$, and as $T_{0} \leq t \leq T, U=U(t, x)$ possesses the exact boundary synchronization shown by (1.10).

The paper is organized as follows: in Section 2 we present the existence and uniqueness of semi-global $C^{2}$ solution to the coupled system of quasilinear wave equations with boundary conditions of various types. Meanwhile, in Section 3 we establish the estimates on the $C^{1}, C^{2}$ norms and the continuity modulus of the second order partial derivatives of $C^{2}$ solutions for a coupled system of nonautonomous linear wave equations. In Section 4, we use a suitable iteration scheme to establish the local exact boundary synchronization for a coupled system of quasilinear wave equations. Some remarks are given in Section 5. Moreover, an appendix is attached at the end for the precise proof of a technical lemma in the main text.

Remark 1.4. Similar results are still valid for the boundary conditions of coupled dissipative type, see Remark 5.2.

Remark 1.5. Precisely speaking, the conditions of $C^{2}$ compatibility at the point $(t, x)=(0, L)$ are given respectively for the boundary conditions (1.6a)-(1.6c) as follows:

$$
\begin{aligned}
& \left\{\begin{array}{l}
\Phi(L)=0, \\
\Psi(L)=0, \\
\Phi^{\prime \prime}(L)=0
\end{array}\right. \\
& \left\{\begin{array}{l}
\Phi^{\prime}(L)=0, \\
\Psi^{\prime}(L)=0 ;
\end{array}\right. \\
& \left\{\begin{array}{l}
\Phi^{\prime}(L)+\bar{B}(\Phi(L)) \Phi(L)=0, \\
\psi_{i}^{\prime}(L)+\sum_{k, j=1}^{N} \frac{\partial \bar{b}_{i j}}{\partial u_{k}}(\Phi(L)) \psi_{k}(L) \varphi_{j}(L)+\sum_{j=1}^{N} \bar{b}_{i j}(\Phi(L)) \psi_{j}(L)=0,
\end{array}\right.
\end{aligned}
$$

where $i=1, \ldots, N$.

Similarly, for any given $H(t)$, the conditions of $C^{2}$ compatibility at the point $(t, x)=(0,0)$ are given by

$$
\begin{aligned}
& \left\{\begin{array}{l}
\Phi(0)=H(0), \\
\Psi(0)=H^{\prime}(0), \\
c^{2}\left(\Phi(0), \Psi(0), \Phi^{\prime}(0)\right) \Phi^{\prime \prime}(0)-A\left(\Phi(0), \Psi(0), \Phi^{\prime}(0)\right) \Phi(0)=H^{\prime \prime}(0) ;
\end{array}\right. \\
& \left\{\begin{array}{l}
\Phi^{\prime}(0)=H(0), \\
\Psi^{\prime}(0)=H^{\prime}(0) ;
\end{array}\right. \\
& \left\{\begin{array}{l}
\Phi^{\prime}(0)-B(\Phi(0)) \Phi(0)=H(0), \\
\psi_{i}^{\prime}(0)-\sum_{k, j=1}^{N} \frac{\partial b_{i j}}{\partial u_{k}}(\Phi(0)) \psi_{k}(0) \varphi_{j}(0)-\sum_{j=1}^{N} b_{i j}(\Phi(0)) \psi_{j}(0)=h_{i}^{\prime}(0),
\end{array}\right.
\end{aligned}
$$

where $i=1, \ldots, N$, respectively. We point out that if some components in $H(t)$ are known in advance, then the conditions of $C^{2}$ compatibility at the point $(t, x)=(0,0)$ involve only those formulas in (1.16) where the corresponding components of $H(t)$ are given. For example, suppose that $h_{1}(t) \equiv 0$, and the other components of $H(t)$ are taken as boundary controls, the conditions of $C^{2}$ compatibility at the point $(t, x)=(0,0)$ are just given by the first formula of (1.16), the right-hand side of which is zero. 
Remark 1.6. For the boundary conditions of Neumann type (1.4b) and (1.6b), noting (1.15b) and (1.16b), the conditions of $C^{2}$ compatibility at the point $(t, x)=(0,0)$ and $(0, L)$ are only related to the initial data and do not depend on the coupled system of wave equations. This fact will play an important role in establishing a unified constructive method independent of the iteration in what follows.

\section{Semi-global Classical solutions for a COUPled System of QuAsilineAR WAVE EQUATIONS}

In order to get the well-posedness of the mixed initial-boundary value problem (1.1), (1.4), (1.6) and (1.7) in the framework of $C^{2}$ solutions, we first reduce the system to a first order quasilinear hyperbolic system, then use the corresponding results of semi-global $C^{1}$ solutions.

Let

$$
V=\left(v_{1}, \ldots, v_{N}\right)^{T}=\frac{\partial U}{\partial x}, W=\left(w_{1}, \ldots, w_{N}\right)^{T}=\frac{\partial U}{\partial t} .
$$

System (1.1) can be reduced to the following first order quasilinear system:

$$
\left\{\begin{array}{l}
\frac{\partial U}{\partial t}=W \\
\frac{\partial V}{\partial t}-\frac{\partial W}{\partial x}=0 \\
\frac{\partial W}{\partial t}-c^{2}(U, W, V) \frac{\partial V}{\partial x}=-A(U, W, V) U .
\end{array}\right.
$$

Let $I_{N}$ be the identity matrix of order $N$. (2.2) can be written in the following matrix form:

$$
\frac{\partial}{\partial t}\left(\begin{array}{c}
U \\
V \\
W
\end{array}\right)+\left(\begin{array}{ccc}
0 & 0 & 0 \\
0 & 0 & -I_{N} \\
0-c^{2}(U, W, V) I_{N} & 0
\end{array}\right) \frac{\partial}{\partial x}\left(\begin{array}{c}
U \\
V \\
W
\end{array}\right)=\left(\begin{array}{c}
W \\
0 \\
-A(U, W, V) U
\end{array}\right) .
$$

The characteristic equation of $(2.2)$ is

$$
\operatorname{det}\left(\begin{array}{ccc}
\lambda I_{N} & 0 & 0 \\
0 & \lambda I_{N} & I_{N} \\
0 & c^{2}(U, W, V) I_{N} & \lambda I_{N}
\end{array}\right)=\lambda^{N}\left|\lambda^{2} I_{N}-c^{2} I_{N}\right|=0
$$

whose solutions, the eigenvalues of system (2.2), are all real:

$$
\lambda_{i}^{-}=-c, \quad \lambda_{i}^{0}=0, \quad \lambda_{i}^{+}=c(i=1, \ldots, N),
$$

and the corresponding left eigenvectors, which constitute a complete set, can be chosen as

$$
l_{i}^{-}=\left(\mathbf{0}, c(U, W, V) \mathbf{e}_{i}, \mathbf{e}_{\mathbf{i}}\right), l_{i}^{0}=\left(\boldsymbol{e}_{\boldsymbol{i}}, \mathbf{0}, \mathbf{0}\right), l_{i}^{+}=\left(\mathbf{0},-c(U, W, V) \mathbf{e}_{\boldsymbol{i}}, \mathbf{e}_{\mathbf{i}}\right) \quad(i=1, \ldots, N),
$$

where $\mathbf{0}=(0, \ldots, 0)$ is the zero vector of order $N$, and $\boldsymbol{e}_{\boldsymbol{i}}=(0, \ldots, \stackrel{(i)}{1}, \ldots, 0)$ is a unit row vector of order $N$. Thus, (2.2) is a first order quasilinear hyperbolic system.

Let

$$
\widetilde{U}=\left(\begin{array}{c}
U \\
V \\
W
\end{array}\right)
$$


and

$$
\left\{\begin{array}{l}
V_{i}^{-}=l_{i}^{-} \widetilde{U}=c v_{i}+w_{i}, \\
V_{i}^{0}=l_{i}^{0} \widetilde{U}=u_{i}, \\
V_{i}^{+}=l_{i}^{+} \widetilde{U}=-c v_{i}+w_{i}
\end{array} \quad(i=1, \ldots, N)\right.
$$

We have

$$
\left\{\begin{array}{l}
V^{-}=c V+W \\
V^{0}=U \\
V^{+}=-c V+W
\end{array}\right.
$$

where $V^{-}=\left(V_{1}^{-}, \ldots, V_{N}^{-}\right)^{T}, V^{0}=\left(V_{1}^{0}, \ldots, V_{N}^{0}\right)^{T}$ and $V^{+}=\left(V_{1}^{+}, \ldots, V_{N}^{+}\right)^{T}$. Then

$$
\left\{\begin{array}{l}
U=V^{0} \\
V=\frac{1}{2 c}\left(V^{-}-V^{+}\right), \\
W=\frac{1}{2}\left(V^{-}+V^{+}\right) .
\end{array}\right.
$$

Under the above transformation, the boundary condition (1.4) can be correspondingly replaced by

$$
\begin{aligned}
& x=0: W=\dot{H}(t), \\
& x=0: V=H(t), \\
& x=0: V-B(U) U=H(t) .
\end{aligned}
$$

Noting (2.10), in a neighborhood of $\widetilde{U}=0$, i.e., $\widetilde{V} \stackrel{\text { def. }}{=}\left(\begin{array}{c}V^{-} \\ V^{0} \\ V^{+}\end{array}\right)=0$, the boundary conditions $(2.11)$ on $x=0$ can be rewritten as

$$
\begin{array}{ll}
x=0: & V^{+}=-V^{-}+2 \dot{H}(t), \\
x=0: & V^{+}=V^{-}-2 c\left(V^{0}, \frac{V^{-}+V^{+}}{2}, H(t)\right) H(t), \\
x=0: & V^{+}=V^{-}-2 c\left(V^{0}, \frac{V^{-}+V^{+}}{2}, B\left(V^{0}\right) V^{0}+H(t)\right)\left(B\left(V^{0}\right) V^{0}+H(t)\right),
\end{array}
$$

which can be uniformly expressed as

$$
x=0: \quad P\left(t, V^{-}, V^{0}, V^{+}\right)=0,
$$

where

$$
\begin{aligned}
& P\left(t, V^{-}, V^{0}, V^{+}\right)=V^{+}+V^{-}-2 \dot{H}(t) \text { for }(2.12 \mathrm{a}), \\
& P\left(t, V^{-}, V^{0}, V^{+}\right)=V^{+}-V^{-}+2 c\left(V^{0}, \frac{V^{-}+V^{+}}{2}, H(t)\right) H(t) \text { for }(2.12 \mathrm{~b}), \\
& P\left(t, V^{-}, V^{0}, V^{+}\right)=V^{+}-V^{-}+2 c\left(V^{0}, \frac{V^{-}+V^{+}}{2}, B\left(V^{0}\right) V^{0}+H(t)\right) \\
& \left(B\left(V^{0}\right) V^{0}+H(t)\right) \\
& \text { for }(2.12 \mathrm{c}) .
\end{aligned}
$$


For the boundary conditions (2.13) on $x=0$, regarding $\left(t, V^{-}, V^{0}, V^{+}\right)$as variables, noting (2.9), under the hypothesis that $\|H\|_{C^{2}}$ (case (1.4a)) or $\|H\|_{C^{1}}$ (cases (1.4b) and (1.4c)) and $\|\widetilde{U}\|_{C^{0}}$ are suitably small (the validity of this hypothesis will be shown by Lem. 2.1 ), it is easy to see that

$$
\operatorname{det}\left(\frac{\partial P_{i}}{\partial V_{j}^{+}}\right) \neq 0, i, j=1, \ldots, N .
$$

Therefore, using the implicit function theorem, for any given $T>0$, on the interval $[0, T]$ the boundary condition on $x=0$ can be locally rewritten around $\widetilde{U}=0$ to the following unified form:

$$
x=0: \quad V^{+}=G\left(t, V^{-}, V^{0}\right)+\widetilde{H}(t),
$$

where $G$ and $\widetilde{H}$ are $C^{1}$ functions with respect to their arguments, and without loss of generality, we may assume that

$$
G(t, 0,0) \equiv 0 .
$$

Obviously, the $C^{1}$ norm of $\widetilde{H}(t)$ is small enough.

Similarly, the boundary conditions $(1.6 \mathrm{a})-(1.6 \mathrm{c})$ on $x=L$ can be also locally rewritten around $\widetilde{U}=0$ to the following unified form:

$$
x=L: \quad V^{-}=\bar{G}\left(V^{0}, V^{+}\right),
$$

in which $\bar{G}$ is a $C^{1}$ function with respect to its arguments and

$$
\bar{G}(0,0) \equiv 0 .
$$

Meanwhile, the initial condition (1.7) can be correspondingly written as

$$
t=0: \widetilde{U}(0, x)=\left(\begin{array}{c}
\Phi(x) \\
\Phi^{\prime}(x) \\
\Psi(x)
\end{array}\right) \stackrel{\text { def. }}{=} \widetilde{U}_{0}(x), 0 \leq x \leq L .
$$

By the conditions of $C^{2}$ compatibility at the points $(t, x)=(0,0)$ and $(0, L)$ for the mixed initial-boundary value problem (1.1), (1.4) and (1.6)-(1.7), it is easy to see that the conditions of $C^{1}$ compatibility at these two points are also satisfied for the mixed initial-boundary value problem (2.2), (2.20), (2.16) and (2.18). Based on the theory of semi-global $C^{1}$ solutions to the first order quasilinear hyperbolic system with zero eigenvalues (see $[5-7,11,13,14,19,20])$, we get immediately the following lemma.

Lemma 2.1. Suppose that on the domain under consideration, $c, a_{i j}, b_{i j}$ and $\bar{b}_{i j}$ are $C^{1}$ functions with respect to their arguments. Suppose furthermore that the conditions of $C^{2}$ compatibility (see (1.15) and (1.16)) are satisfied at the points $(t, x)=(0,0)$ and $(0, L)$, respectively. For any given and possibly quite large $T>0$, the forward mixed initial-boundary value problem (1.1), (1.4) and (1.6)-(1.7) admits a unique $C^{2}$ solution $U=U(t, x)$ with small $C^{2}$ norm on the domain

$$
R(T)=\{(t, x) \mid 0 \leq t \leq T, 0 \leq x \leq L\},
$$

provided that $\|(\Phi, \Psi)\|_{\left(C^{2}[0, L]\right)^{N} \times\left(C^{1}[0, L]\right)^{N}}$ and $\|H\|_{\left(C^{2}[0, T]\right)^{N}}$ (case (1.4a)) or $\|H\|_{\left(C^{1}[0, T]\right)^{N}}$ (cases (1.4b)-(1.4c)) are suitably small (possibly depending on $T$ ). More precisely, for any given $\varepsilon>0$ small enough, if

$$
\|(\Phi, \Psi)\|_{\left(C^{2}[0, L]\right)^{N} \times\left(C^{1}[0, L]\right)^{N}} \leq \varepsilon
$$


and

$$
\|H\|_{\left(C^{2}[0, T]\right)^{N}}(\text { case }(1.4 \mathrm{a})) \text { or }\|H\|_{\left(C^{1}[0, T]\right)^{N}}(\text { cases }(1.4 \mathrm{~b})-(1.4 \mathrm{c})) \leq \varepsilon \text {, }
$$

then

$$
\|U\|_{\left(C^{2}[R(T)]\right)^{N}} \leq G(\varepsilon),
$$

where $G(\varepsilon)$ is a $C^{0}$ function of $\varepsilon>0$, satisfying $G(0)=0$.

\section{Estimates on the $C^{1}, C^{2}$ NORMS of SOlutions And THE CONTINuity Modulus OF THE SECOND ORDER PARTIAL DERIVATIVES OF SOLUTIONS FOR A COUPLED SYSTEM OF NONAUTONOMOUS LINEAR WAVE EQUATIONS}

In order to obtain the exact boundary synchronization for the quasilinear system (1.1), (1.4) and (1.6), we have to investigate the $C^{1}, C^{2}$ norms of solutions and the continuity modulus of the second order partial derivatives of solutions for a coupled system of nonautonomous linear wave equations. Here, the continuity modulus of a function $f(t, x)$ on $R(T)=\{(t, x) \mid 0 \leq t \leq T, 0 \leq x \leq L\}$ is the following non-negative function:

$$
\omega(\eta)=\omega(\eta \mid f) \stackrel{\text { def. }}{=} \sup _{\substack{\left|t^{\prime}-t^{\prime \prime}\right| \leq \eta,\left|x^{\prime}-x^{\prime \prime}\right| \leq \eta \\\left(t^{\prime}, x^{\prime}\right),\left(t^{\prime \prime}, x^{\prime \prime}\right) \in R(T)}}\left|f\left(t^{\prime}, x^{\prime}\right)-f\left(t^{\prime \prime}, x^{\prime \prime}\right)\right|, \quad \eta \geq 0 .
$$

Similarly, the continuity modulus of a vector function $f=\left(f_{1}, \ldots f_{n}\right)$ can be defined by

$$
\omega(\eta \mid f) \stackrel{\text { def. }}{=} \max _{i=1, \ldots, n} \omega\left(\eta \mid f_{i}\right),
$$

and the continuity modulus of a matrix function $A=\left(a_{i j}\right)_{n \times n}$ can be defined by

$$
\omega(\eta \mid A) \stackrel{\text { def. }}{=} \max _{i, j=1, \ldots, n} \omega\left(\eta \mid a_{i j}\right) .
$$

In this section, we still denote $U=U(t, x)$ as the unknown vector function, $c=c(t, x)$ as the common propagation speed and $A=A(t, x), B=B(t)$ and $\bar{B}=\bar{B}(t)$ as coupling matrices. Consider the following coupled system of nonautonomous wave equations:

$$
\frac{\partial^{2} U}{\partial t^{2}}-c^{2}(t, x) \frac{\partial^{2} U}{\partial x^{2}}+A(t, x) U=F(t, x)
$$

where $U=\left(u_{1}, \ldots, u_{N}\right)^{T}$ is the unknown vector function of $(t, x), A(t, x)=\left(a_{i j}(t, x)\right)$ is an $N \times N$ matrix with $C^{1}$ elements, $c(t, x)>0$, the common propagation speed for the coupled system, is a $C^{1}$ function of $(t, x)$, while $F(t, x)=\left(f_{1}(t, x), \ldots, f_{N}(t, x)\right)^{T}$ is also a $C^{1}$ vector function of $(t, x)$.

At the end $x=0$, corresponding to (1.4a) $-(1.4 \mathrm{c})$, we prescribe any one of the following boundary conditions:

$$
\begin{aligned}
& x=0: U=H(t) \text { (Dirichlet type), } \\
& x=0: U_{x}=H(t) \text { (Neumann type), } \\
& x=0: U_{x}-B(t) U=H(t) \text { (Coupled third type), }
\end{aligned}
$$

in which $B(t)=\left(b_{i j}(t)\right)$ is an $N \times N$ matrix with $C^{1}$ elements, and $H(t)=\left(h_{1}(t), \ldots, h_{N}(t)\right)^{T}$ are $C^{2}$ (case (3.5a)) or $C^{1}$ (cases (3.5b) and (3.5c)) functions of $t$. 
At the end $x=L$, corresponding to (1.6a)-(1.6c), we prescribe any one of the following boundary conditions:

$$
\begin{aligned}
& x=L: U=\bar{H}(t) \text { (Dirichlet type), } \\
& x=L: U_{x}=\bar{H}(t)(\text { Neumann type), } \\
& x=L: U_{x}+\bar{B}(t) U=\bar{H}(t) \text { (Coupled third type), }
\end{aligned}
$$

in which $\bar{B}(t)=\left(\bar{b}_{i j}(t)\right)$ is an $N \times N$ matrix with $C^{1}$ elements, and $\bar{H}(t)=\left(\bar{h}_{1}(t), \ldots, \bar{h}_{N}(t)\right)^{T}$ are $C^{2}$ (case (3.6a)) or $C^{1}$ (cases (3.6b) and (3.6c)) functions of $t$.

Denote

$$
\begin{aligned}
l & = \begin{cases}1 & \text { case }(3.5 \mathrm{a}), \\
0 & \text { cases }(3.5 \mathrm{~b})-(3.5 \mathrm{c}),\end{cases} \\
s & =l+1, \\
& \bar{l}= \begin{cases}1 & \text { case }(3.6 \mathrm{a}), \\
0 & \text { cases }(3.6 \mathrm{~b})-(3.6 \mathrm{c}),\end{cases}
\end{aligned}
$$

Suppose that on the domain under consideration, we have

$$
\|c\|_{1}+\|A\|_{1}+\|B\|_{1}+\|\bar{B}\|_{1} \leq D<+\infty
$$

where $D$ is a positive constant. Here and hereafter, for simplicity we denote $\|\cdot\|_{0},\|\cdot\|_{1}$ and $\|\cdot\|_{2}$ as as the corresponding $C^{0}, C^{1}$ and $C^{2}$ norms, respectively.

For the mixed initial-boundary value problem (3.4), (3.5), (3.6) and (1.7), by the estimates established in $[6,7]$ for $C^{1}$ and $C^{2}$ norms of solutions, as well as the continuity modulus of the second order partial derivatives of solutions, obtained by a similar manner, we have

Lemma 3.1. For any given $T>0$, suppose that $c, a_{i j}, b_{i j}, \bar{b}_{i j}$ and $f_{i}(i, j=1, \ldots, N)$ are all $C^{1}$ functions on the domain $R(T)=\{(t, x) \mid 0 \leq t \leq T, 0 \leq x \leq L\}$. Suppose furthermore that the conditions of $C^{2}$ compatibility are satisfied at the points $(t, x)=(0,0)$ and $(0, L)$, respectively. Then the forward mixed initial-boundary value problem (3.4)-(3.6) and (1.7) admits a unique $C^{2}$ solution $U=U(t, x)$ on the domain $R(T)=\{(t, x) \mid 0 \leq t \leq$ $T, 0 \leq x \leq L\}$, and we have

$$
\|U\|_{\left(C^{1}[R(T)]\right)^{N}} \leq C_{1}\left(\|(\Phi, \Psi)\|_{\left(C^{1}[0, L]\right)^{N} \times\left(C^{0}[0, L]\right)^{N}}+\|(H, \bar{H})\|_{\left(C^{l}[0, T]\right)^{N} \times\left(C^{\bar{l}}[0, T]\right)^{N}}+\|F\|_{\left(C^{0}[R(T)]\right)^{N}}\right)
$$

and

$$
\|U\|_{\left(C^{2}[R(T)]\right)^{N}} \leq C_{2}\left(\|(\Phi, \Psi)\|_{\left(C^{2}[0, L]\right)^{N} \times\left(C^{1}[0, L]\right)^{N}}+\|(H, \bar{H})\|_{\left(C^{s}[0, T]\right)^{N} \times\left(C^{\bar{s}}[0, T]\right)^{N}}+\|F\|_{\left(C^{1}[R(T)]\right)^{N}}\right),
$$

where $C_{1}=C_{1}(T, D)$ and $C_{2}=C_{2}(T, D)$ are positive constants depending only on $T$ and $D$. Moreover, if

$$
F(t, x) \equiv 0,
$$

then for any given $\eta$ with

$$
0<\eta \leq \min _{(t, x) \in R(T)} \frac{L}{c(t, x)}
$$

we have

$$
\omega\left(\eta \mid \frac{\partial^{2} U}{\partial t^{2}}\right)+\omega\left(\eta \mid \frac{\partial^{2} U}{\partial x^{2}}\right)+\omega\left(\eta \mid \frac{\partial^{2} U}{\partial t \partial x}\right) \leq \Omega(\eta)
$$


where

$$
\begin{aligned}
\Omega(\eta)= & C_{3}\left(\omega\left(\eta \mid \Phi_{x x}\right)+\omega\left(\eta \mid \Psi_{x}\right)+\omega\left(\eta \mid \frac{d^{s} H}{d t^{s}}\right)+\omega\left(\eta \mid \frac{d^{\bar{s}} \bar{H}}{d t^{\bar{s}}}\right)+\left(\|\Phi\|_{2}+\|\Psi\|_{1}+\|H\|_{s}+\|\bar{H}\|_{\bar{s}}\right)\right. \\
& \left.\cdot\left(\eta+\omega\left(\eta \mid c_{x}\right)+\omega\left(\eta \mid c_{t}\right)+\omega\left(\eta \mid A_{x}\right)+\omega\left(\eta \mid B_{t}\right)+\omega\left(\eta \mid \bar{B}_{t}\right)\right)\right)
\end{aligned}
$$

and $C_{3}=C_{3}(T, D)$ is a positive constant depending on $T$ and $D$.

Remark 3.2. For the backward mixed initial-boundary value problem (3.4)-(3.6) with the final condition

$$
t=T:\left(U, U_{t}\right)=(\bar{\Phi}(x), \bar{\Psi}(x)), 0 \leq x \leq L,
$$

satisfying the conditions of $C^{2}$ compatibility at the points $(t, x)=(T, 0)$ and $(T, L)$, respectively, similar results can be obtained.

\section{Proof of Theorem 1.1}

Arbitrarily choose $H^{(0)}(t)$ (in which $\left.h_{1}^{(0)}(t) \equiv 0\right)$ to be a $C^{2}$ (case (1.4a)) or $C^{1}$ (cases $\left.(1.4 \mathrm{~b})-(1.4 \mathrm{c})\right)$ vector function of $t$, satisfying

$$
\left\|H^{(0)}\right\|_{\left(C^{s}[0, T]\right)^{N}} \leq \varepsilon
$$

where

$$
s=\left\{\begin{array}{l}
2, \text { case }(1.4 \mathrm{a}), \\
1, \text { cases }(1.4 \mathrm{~b})-(1.4 \mathrm{c}),
\end{array}\right.
$$

and $\varepsilon>0$, given by (1.8), is a suitably small positive constant, such that the conditions of $C^{2}$ compatibility are satisfied at the point $(t, x)=(0,0)$, and

$$
H^{(0)}(t) \equiv 0, \quad T_{0} \leq t \leq T
$$

Remark 4.1. Since we have assumed $h_{1}^{(0)}(t) \equiv 0$, by the conditions of $C^{2}$ compatibility $(c f$. (1.15)) at the point $(t, x)=(0,0)$, the initial condition (1.7) can not be arbitrarily given near $x=0$. Although this is a special case in Theorem 1.3, it is not an issue for the generality of the whole proof.

By Lemma 2.1, the initial-boundary value problem (1.1), (1.4) (in which $H(t)=H^{(0)}(t)$ ) and (1.6)-(1.7) admits a unique $C^{2}$ solution $U=U^{(0)}(t, x)$ on the domain $R(T)=\{(t, x) \mid 0 \leq t \leq T, 0 \leq x \leq L\}$, satisfying

$$
\left\|U^{(0)}\right\|_{\left(C^{2}[R(T)]\right)^{N}} \leq G(\varepsilon),
$$

where $G(\varepsilon)$ is a $C^{0}$ function of $\varepsilon \geq 0$, satisfying

$$
G(0)=0 .
$$

Since $U^{(0)} \in C^{2}$, there exists a non-negative function $\Omega_{0}(\eta)$ of $\eta\left(\Omega_{0}(\eta) \rightarrow 0\right.$ as $\left.\eta \rightarrow 0\right)$, such that

$$
\omega\left(\eta \mid \frac{\partial^{2} U^{(0)}}{\partial t^{2}}\right)+\omega\left(\eta \mid \frac{\partial^{2} U^{(0)}}{\partial x^{2}}\right)+\omega\left(\eta \mid \frac{\partial^{2} U^{(0)}}{\partial t \partial x}\right) \leq \Omega_{0}(\eta) .
$$

Here we point out that, being the first step of the iteration, $U^{(0)}$ is not asked to be synchronized for $T_{0} \leq t \leq T$. 
Let

$$
U^{(-1)}(t, x) \stackrel{\text { def. }}{=} U^{(0)}(t, x) .
$$

For any given $m \geq 0$, we use the following iteration procedure to get $U^{(m)}(t, x)$ from $U^{(m-1)}(t, x)$ on $R(T)=$ $\{(t, x) \mid 0 \leq t \leq T, 0 \leq x \leq L\}$ :

$$
\frac{\partial^{2} U^{(m)}}{\partial t^{2}}-c^{2}\left(U^{(m-1)}, U_{t}^{(m-1)}, U_{x}^{(m-1)}\right) \frac{\partial^{2} U^{(m)}}{\partial x^{2}}+A\left(U^{(m-1)}, U_{t}^{(m-1)}, U_{x}^{(m-1)}\right) U^{(m)}=0,
$$

the boundary condition on $x=0$ is given by any one of

$$
\begin{aligned}
& x=0: U^{(m)}=H^{(m)}(t), \\
& x=0: U_{x}^{(m)}=H^{(m)}(t), \\
& x=0: U_{x}^{(m)}-B\left(U^{(m-1)}\right) U^{(m)}=H^{(m)}(t),
\end{aligned}
$$

the boundary condition on $x=L$ is given by any one of

$$
\begin{aligned}
& x=L: U^{(m)}=0, \\
& x=L: U_{x}^{(m)}=0, \\
& x=L: U_{x}^{(m)}-\bar{B}\left(U^{(m-1)}\right) U^{(m)}=0,
\end{aligned}
$$

and the initial condition is

$$
t=0:\left(U^{(m)}, U_{t}^{(m)}\right)=(\Phi(x), \Psi(x)), 0 \leq x \leq L .
$$

In this iteration scheme, for $m=0$, since $H^{(0)}(t)$ has been chosen, the corresponding solution is just $U^{(0)}(t, x)$; while, for each $m \geq 1, H^{(m)}(t)$ is a $C^{2}$ (case (4.9a)) or $C^{1}$ (cases (4.9b) and (4.9c)) function of $t$ to be determined. Our goal is to find a suitable $H^{(m)}(t)$ (in which $h_{1}^{(m)}(t) \equiv 0$ ) for each $m \geq 1$, such that the corresponding mixed initial-boundary value problem (4.8)-(4.11) admits a $C^{2}$ solution $U^{(m)}=U^{(m)}(t, x)$ on the domain $R(T)=\{(t, x) \mid 0 \leq t \leq T, 0 \leq x \leq L\}$, satisfying the exact boundary synchronization (1.10). Notice that in the whole iteration procedure, the form of the conditions of $C^{2}$ compatibility (1.15)-(1.16) at the points $(t, x)=(0,0)$ and $(0, L)$ never changes (just replacing $H(t)$ by $\left.H^{(m)}(t)\right)$.

If the synchronization sequence $\left\{U^{(m)}(t, x)\right\}(m \geq 1)$ is obtained by means of a sequence $\left\{H^{(m)}(t)\right\}$ (in which $h_{1}^{(m)}(t) \equiv 0(0 \leq t \leq T)$ for $\left.m \geq 1\right)$ of suitable boundary controls, and if one can prove that

$$
U^{(m)}(t, x) \rightarrow U(t, x) \text { in }\left(C^{2}[R(T)]\right)^{N} \text { as } m \rightarrow+\infty,
$$

then $U(t, x)$ should satisfy (1.1) and (1.6)-(1.7) and possesses the synchronization (1.10), and the boundary control corresponding to (1.4) is given by

$$
H(t)=\lim _{m \rightarrow+\infty} H^{(m)}(t)
$$

with $h_{1}(t) \equiv 0(0 \leq t \leq T)$. Therefore, in order to get Theorem 1.1, it suffices to establish the following.

Lemma 4.2. Under the assumptions of Theorem 1.1, for any given integer $m \geq 0$, there exist a boundary control $H^{(m)}(t)$ (in which $\left.h_{1}^{(m)}(t) \equiv 0(0 \leq t \leq T)\right)$ and positive constants $C_{4}=C_{4}\left(T_{0}, T\right), C_{5}=C_{5}\left(T_{0}, T\right)$ and $C_{6}=C_{6}\left(T_{0}, T\right)$, such that the mixed initial-boundary value problem (4.8)-(4.11) admits a unique $C^{2}$ solution $U^{(m)}=U^{(m)}(t, x)$ on the domain $R(T)=\{(t, x) \mid 0 \leq t \leq T, 0 \leq x \leq L\}$, which satisfies

$$
\begin{aligned}
& \left\|U^{(m)}\right\|_{\left(C^{2}[R(T)]\right)^{N}} \leq C_{4}(G(\varepsilon)+\varepsilon), \\
& \left\|U^{(m)}-U^{(m-1)}\right\|_{\left(C^{1}[R(T)]\right)^{N} \leq C_{5}^{m}(G(\varepsilon)+\varepsilon)^{m},} \\
& \omega\left(\eta \mid \frac{\partial^{2} U^{(m)}}{\partial t^{2}}\right)+\omega\left(\eta \mid \frac{\partial^{2} U^{(m)}}{\partial x^{2}}\right)+\omega\left(\eta \mid \frac{\partial^{2} U^{(m)}}{\partial t \partial x}\right) \leq \Omega_{1}(\eta),
\end{aligned}
$$


where

$$
\Omega_{1}(\eta)=\frac{C_{6}\left(\omega\left(\eta \mid \Phi_{x x}\right)+\omega\left(\eta \mid \Psi_{x}\right)+\Omega_{0}(\eta)+(G(\varepsilon)+\varepsilon)\left(\eta+\omega\left(\eta \mid \Gamma^{*}\right)\right)\right)}{1-C_{6}(G(\varepsilon)+\varepsilon)},
$$

while $G(\varepsilon)$ and $\Omega_{0}(\eta)$ are given by (4.4) and (4.6), and

$$
\Gamma^{*}=\left\{\frac{\partial c}{\partial u_{k}}, \frac{\partial c}{\partial u_{k t}}, \frac{\partial c}{\partial u_{k x}}, \frac{\partial a_{i j}}{\partial u_{k}}, \frac{\partial a_{i j}}{\partial u_{k t}}, \frac{\partial a_{i j}}{\partial u_{k x}}, \frac{\partial b_{i j}}{\partial u_{k}}, \frac{\partial \bar{b}_{i j}}{\partial u_{k}} ; i, j, k=1, \ldots, N\right\} .
$$

Moreover, for any given $m \geq 1, U^{(m)}(t, x)$ possesses the synchronization (1.10) for $T_{0} \leq t \leq T$.

Proof. For $m=0$, since $H^{(0)}(t)$ has been determined, taking $C_{4} \geq 1$, (4.4) immediately yields (4.14). Moreover, we have (4.15) because of (4.7). Combining (4.6) and (4.17), one gets (4.16), provided that $\varepsilon>0$ is suitably small and $C_{6} \geq 1$.

By induction, suppose that for a given $m \geq 1$ we have

$$
\begin{aligned}
& \left\|U^{(m-1)}\right\|_{\left(C^{2}[R(T)]\right)^{N}} \leq C_{4}(G(\varepsilon)+\varepsilon), \\
& \left\|U^{(m-1)}-U^{(m-2)}\right\|_{\left(C^{1}[R(T)]\right)^{N} \leq C_{5}^{m-1}}(G(\varepsilon)+\varepsilon)^{m-1}, \\
& \omega\left(\eta \mid \frac{\partial^{2} U^{(m-1)}}{\partial t^{2}}\right)+\omega\left(\eta \mid \frac{\partial^{2} U^{(m-1)}}{\partial x^{2}}\right)+\omega\left(\eta \mid \frac{\partial^{2} U^{(m-1)}}{\partial t \partial x}\right) \leq \Omega_{1}(\eta) .
\end{aligned}
$$

Then for this $m$, let

$$
\left\{\begin{array}{l}
w_{i}^{(m)}=u_{i+1}^{(m)}-u_{i}^{(m)}, \\
\widetilde{h}_{i}^{(m)}=h_{i+1}^{(m)}-h_{i}^{(m)}
\end{array} \quad i=1, \ldots, N-1 .\right.
$$

Thanks to the assumption (1.11), it is easy to see that from the original system (4.8) for the variable $U^{(m)}=$ $\left(u_{1}^{(m)}, \ldots, u_{N}^{(m)}\right)$, we can get the following system for the variable $W^{(m)}=\left(w_{1}^{(m)}, \ldots, w_{N-1}^{(m)}\right)$ (see also $\left.[14]\right)$ :

$$
\frac{\partial^{2} W^{(m)}}{\partial t^{2}}-c^{2}\left(U^{(m-1)}, U_{t}^{(m-1)}, U_{x}^{(m-1)}\right) \frac{\partial^{2} W^{(m)}}{\partial x^{2}}+\widetilde{A}\left(U^{(m-1)}, U_{t}^{(m-1)}, U_{x}^{(m-1)}\right) W^{(m)}=0,
$$

where $\widetilde{A}=\left(\widetilde{a}_{i j}\right)$ is an $(N-1) \times(N-1)$ reduced matrix with

$$
\begin{aligned}
& \tilde{a}_{i j}\left(U^{(m-1)}, U_{t}^{(m-1)}, U_{x}^{(m-1)}\right) \\
= & \sum_{p=j+1}^{N}\left(a_{i+1, p}\left(U^{(m-1)}, U_{t}^{(m-1)}, U_{x}^{(m-1)}\right)-a_{i p}\left(U^{(m-1)}, U_{t}^{(m-1)}, U_{x}^{(m-1)}\right)\right) \\
= & \sum_{p=1}^{j}\left(a_{i, p}\left(U^{(m-1)}, U_{t}^{(m-1)}, U_{x}^{(m-1)}\right)-a_{i+1, p}\left(U^{(m-1)}, U_{t}^{(m-1)}, U_{x}^{(m-1)}\right)\right), i, j=1, \ldots, N-1 .
\end{aligned}
$$

Similarly, thanks to the assumptions (1.12)-(1.13), from the original boundary conditions (4.9)-(4.10) for the variable $U^{(m)}$, we can get, respectively, the boundary conditions for the variable $W^{(m)}$ :

$$
\begin{aligned}
& x=0: W^{(m)}=\widetilde{H}^{(m)}(t), \\
& x=0: W_{x}^{(m)}=\widetilde{H}^{(m)}(t), \\
& x=0: W_{x}^{(m)}-\widetilde{B}\left(U^{(m-1)}\right) W^{(m)}=\widetilde{H}^{(m)}(t)
\end{aligned}
$$


and

$$
\begin{aligned}
& x=L: W^{(m)}=0, \\
& x=L: W_{x}^{(m)}=0, \\
& x=L: W_{x}^{(m)}+\widetilde{\bar{B}}\left(U^{(m-1)}\right) W^{(m)}=0,
\end{aligned}
$$

in which $\widetilde{H}^{(m)}(t)=\left(\tilde{h}_{1}^{(m)}(t), \ldots, \tilde{h}_{N-1}^{(m)}(t)\right)^{T}$, and $\widetilde{B}=\left(\widetilde{b}_{i j}\right)$ and $\widetilde{\bar{B}}=\left(\widetilde{\bar{b}}_{i j}\right)$ are $(N-1) \times(N-1)$ matrices reduced by $B=\left(b_{i j}\right)$ and $\bar{B}=\left(\bar{b}_{i j}\right)$ in the following way:

$$
\begin{aligned}
\tilde{b}_{i j}\left(U^{(m-1)}\right) & =\sum_{p=j+1}^{n}\left(b_{i+1, p}\left(U^{(m-1)}\right)-b_{i p}\left(U^{(m-1)}\right)\right) \\
& =\sum_{p=1}^{j}\left(b_{i, p}\left(U^{(m-1)}\right)-b_{i+1, p}\left(U^{(m-1)}\right)\right), i, j=1, \ldots, N-1
\end{aligned}
$$

etc.

The initial condition of $W^{(m)}$ is given by

$$
t=0:\left(W^{(m)}, W_{t}^{(m)}\right)=(\widetilde{\Phi}(x), \widetilde{\Psi}(x)), 0 \leq x \leq L,
$$

where

$$
\left\{\begin{array}{l}
\widetilde{\Phi}(x)=\left(\varphi_{2}(x)-\varphi_{1}(x), \ldots, \varphi_{N}(x)-\varphi_{N-1}(x)\right)^{T}, \\
\widetilde{\Psi}(x)=\left(\psi_{2}(x)-\psi_{1}(x), \ldots, \psi_{N}(x)-\psi_{N-1}(x)\right)^{T} .
\end{array}\right.
$$

Moreover, it easily follows from the corresponding conditions of $C^{2}$ compatibility at the point $(t, x)=(0, L)$ for $U^{(m)}$ that the conditions of $C^{2}$ compatibility at the point $(t, x)=(0, L)$ for $W^{(m)}$ are still satisfied.

Noting (4.22), once we achieve the exact null controllability for $W^{(m)}$, we certainly have the exact synchronization for $U^{(m)}$. Similar to the method in $[2,10]$, our next step is to construct a $C^{2}$ solution $W^{(m)}=W^{(m)}(t, x)$ on $R(T)=\{(t, x) \mid 0 \leq t \leq T, 0 \leq x \leq L\}$ for each $m \geq 1$, satisfying simultaneously the system (4.23), the initial condition (4.28), the null final condition

$$
W^{(m)}(t, x) \equiv 0, \quad T_{0} \leq t \leq T
$$

and the boundary condition (4.26) on $x=L$. To this end, we need a suitable treatment on the constructive method for realizing the exact boundary null controllability of $W^{(m)}$. The key point in the proof is that the artificial boundary conditions on $x=0$ in the constructive method should be independent of the iteration (4.23). For this purpose, noting the conditions of $C^{2}$ compatibility at the point $(t, x)=(0,0)(c f$. (1.15)), we will take artificial boundary conditions of Neumann type on $x=0$, which is independent of $m$, instead of the commonly used artificial boundary conditions of Dirichlet type (see Rems. 1.5-1.6). Thus, for the sequence $\left\{W^{(m)}\right\}$, we can prove the following Lemma 4.3 (for the details of its proof, see Appendix).

Lemma 4.3. For any given $m \geq 1$, under the assumptions of Theorem 1.1, there exist a boundary control $\widetilde{H}^{(m)}(t)$ on $x=0$, composed of $C^{2}$ (case $\left.(4.25 \mathrm{a})\right)$ or $C^{1}$ (cases $\left.(4.25 \mathrm{~b})-(4.25 \mathrm{c})\right)$ functions of $t$ with support on $\left[0, T_{0}\right]$, and positive constants $C_{7}\left(T_{0}, T\right), C_{8}\left(T_{0}, T\right)$ and $C_{9}\left(T_{0}, T\right)$, such that the mixed initial-boundary value problem (4.23), (4.25)-(4.26) and (4.28) admits a unique $C^{2}$ solution $W^{(m)}=W^{(m)}(t, x)$ on the domain $R(T)=$ $\{(t, x) \mid 0 \leq t \leq T, 0 \leq x \leq L\}$, satisfying (4.30) and

$$
\begin{aligned}
& \left\|W^{(m)}\right\|_{\left(C^{2}[R(T)]\right)^{N-1}} \leq C_{7}(G(\varepsilon)+\varepsilon), \\
& \left\|W^{(m)}-W^{(m-1)}\right\|_{\left(C^{1}[R(T)]\right)^{N-1}} \leq C_{8} C_{5}^{m-1}(G(\varepsilon)+\varepsilon)^{m}
\end{aligned}
$$


and

$$
\omega\left(\eta \mid \frac{\partial^{2} W^{(m)}}{\partial t^{2}}\right)+\omega\left(\eta \mid \frac{\partial^{2} W^{(m)}}{\partial x^{2}}\right)+\omega\left(\eta \mid \frac{\partial^{2} W^{(m)}}{\partial t \partial x}\right) \leq \Omega_{2}(\eta)
$$

where $C_{5}$ is given by Lemma 4.1,

$$
\Omega_{2}(\eta)=C_{9}\left(\omega\left(\eta \mid \Phi_{x x}\right)+\omega\left(\eta \mid \Psi_{x}\right)+\Omega_{0}(\eta)+(G(\varepsilon)+\varepsilon)\left(\eta+\Omega_{1}(\eta)+\Omega\left(\eta \mid \Gamma^{*}\right)\right)\right),
$$

and $G(\varepsilon), \Omega_{0}(\eta)$ and $\Omega_{1}(\eta)$ are given by (4.4), (4.6) and (4.17), respectively.

Noting (4.31)-(4.33), by the way to obtain the corresponding boundary controls in the constructive method (see [10]), we have

$$
\begin{aligned}
& \left\|\widetilde{H}^{(m)}\right\|_{\left(C^{e}[0, T]\right)^{N-1}} \leq C_{10}\left\|W^{(m)}\right\|_{\left(C^{2}[R(T)]\right)^{N-1}} \leq C_{11}(G(\varepsilon)+\varepsilon), \\
& \left\|\widetilde{H}^{(m)}-\widetilde{H}^{(m-1)}\right\|_{\left(C^{\bar{e}}[0, T]\right)^{N-1}} \leq C_{12}\left\|W^{(m)}-W^{(m-1)}\right\|_{\left(C^{1}[R(T)]\right)^{N-1}} \leq C_{13} C_{5}^{m-1}(G(\varepsilon)+\varepsilon)^{m}
\end{aligned}
$$

and

$$
\omega\left(\eta \mid \frac{\mathrm{d}^{e} \widetilde{H}^{(m)}}{\mathrm{d} t^{e}}\right) \leq C_{14} \Omega_{2}(\eta)
$$

where

$$
e=\left\{\begin{array}{ll}
2, & \text { case }(4.25 \mathrm{a}), \\
1, & \text { cases }(4.25 \mathrm{~b})--(4.25 \mathrm{c}),
\end{array} \quad \bar{e}= \begin{cases}1, & \text { case }(4.25 \mathrm{a}), \\
0, & \text { cases }(4.25 \mathrm{~b})--(4.25 \mathrm{c}) .\end{cases}\right.
$$

and $C_{10}, C_{11}, C_{12}, C_{13}$ and $C_{14}$ are all positive constants depending only on $T_{0}$ and $T$.

Thus, by (4.22) and noting $h_{1}^{(m)} \equiv 0$, we get

$$
\begin{aligned}
& \left\|H^{(m)}\right\|_{\left(C^{r}[R(T)]\right)^{N}} \leq C_{15}(G(\varepsilon)+\varepsilon), \\
& \left\|H^{(m)}-H^{(m-1)}\right\|_{\left(C^{\top}[R(T)]\right)^{N}} \leq C_{16} C_{5}^{m-1}(G(\varepsilon)+\varepsilon)^{m}
\end{aligned}
$$

and

$$
\omega\left(\eta \mid \frac{\mathrm{d}^{r} H^{(m)}}{\mathrm{d} t^{r}}\right) \leq C_{17} \Omega_{2}(\eta)
$$

where

$$
r=\left\{\begin{array}{ll}
2, & \text { case }(4.9 \mathrm{a}), \\
1, & \text { cases }(4.9 \mathrm{~b})-(4.9 \mathrm{c}),
\end{array} \quad \bar{r}= \begin{cases}1, & \text { case }(4.9 \mathrm{a}) \\
0, & \text { cases }(4.9 \mathrm{~b}) \text { and }(4.9 \mathrm{c})\end{cases}\right.
$$

and $C_{15}, C_{16}, C_{17}$ are positive constants depending only on $T_{0}$ and $T$.

Obviously, for $m \geq 1$, the previously obtained boundary control $H^{(m)}$ satisfies the conditions of $C^{2}$ compatibility at the points $(t, x)=(0,0)$ and $(0, L)$ for the mixed initial-boundary value problem (4.8)-(4.11), respectively. Noticing $(4.21),(4.39)$ and $(4.41)$, it is easy to see from Lemma 3.1 that

$$
\left\|U^{(m)}\right\|_{\left(C^{2}[R(T)]\right)^{N}} \leq C_{18}(G(\varepsilon)+\varepsilon)
$$


and

$$
\begin{aligned}
& \omega\left(\eta \mid \frac{\partial^{2} U^{(m)}}{\partial t^{2}}\right)+\omega\left(\eta \mid \frac{\partial^{2} U^{(m)}}{\partial x^{2}}\right)+\omega\left(\eta \mid \frac{\partial^{2} U^{(m)}}{\partial t \partial x}\right) \\
& \leq C_{19}\left(\omega\left(\eta \mid \Phi_{x x}\right)+\omega\left(\eta \mid \Psi_{x}\right)+\Omega_{0}(\eta)+(G(\varepsilon)+\varepsilon)\left(\eta+\Omega_{1}(\eta)+\Omega\left(\eta \mid \Gamma^{*}\right)\right)\right),
\end{aligned}
$$

where $C_{18}$ and $C_{19}$ are positive constants depending only on $T$ and $T_{0}$. Therefore, taking $C_{4} \geq C_{18}, C_{6} \geq C_{19}$, we get (4.14) and (4.16).

On the other hand, let

$$
V^{(m)}=U^{(m)}-U^{(m-1)} .
$$

$V^{(m)}$ satisfies the following mixed initial-boundary value problem:

$$
\begin{aligned}
& \frac{\partial^{2} V^{(m)}}{\partial t^{2}}-c^{2}\left(U^{(m-1)}, U_{t}^{(m-1)}, U_{x}^{(m-1)}\right) \frac{\partial^{2} V^{(m)}}{\partial x^{2}}+A\left(U^{(m-1)}, U_{t}^{(m-1)}, U_{x}^{(m-1)}\right) V^{(m)} \\
& +\left(c^{2}\left(U^{(m-1)}, U_{t}^{(m-1)}, U_{x}^{(m-1)}\right)-c^{2}\left(U^{(m-2)}, U_{t}^{(m-2)}, U_{x}^{(m-2)}\right)\right) \frac{\partial^{2} U^{(m-1)}}{\partial x^{2}} \\
& +\left(A\left(U^{(m-1)}, U_{t}^{(m-1)}, U_{x}^{(m-1)}\right)-A\left(U^{(m-2)}, U_{t}^{(m-2)}, U_{x}^{(m-2)}\right)\right) U^{(m-1)}=0
\end{aligned}
$$

with the initial condition

$$
t=0:\left(V^{(m)}, V_{t}^{(m)}\right)=(0,0), 0 \leq x \leq L
$$

and the boundary conditions

$$
\begin{aligned}
& x=0: V^{(m)}=H^{(m)}-H^{(m-1)}, \\
& x=0: V_{x}^{(m)}=H^{(m)}-H^{(m-1)}, \\
& x=0: V_{x}^{(m)}-B\left(U^{(m-1)}\right) V^{(m)}=\left(B\left(U^{(m-1)}\right)-B\left(U^{(m-2)}\right)\right) U^{(m-1)}+H^{(m)}-H^{(m-1)}
\end{aligned}
$$

and

$$
\begin{aligned}
& x=L: V^{(m)}=0, \\
& x=L: V_{x}^{(m)}=0, \\
& x=L: V_{x}^{(m)}+\bar{B}\left(U^{(m-1)}\right) V^{(m)}=\left(\bar{B}\left(U^{(m-2)}\right)-\bar{B}\left(U^{(m-1)}\right)\right) U^{(m-1)} .
\end{aligned}
$$

Noting (4.19)-(4.20), we have

$$
\begin{aligned}
& \left\|\left(A\left(U^{(m-1)}, U_{t}^{(m-1)}, U_{x}^{(m-1)}\right)-A\left(U^{(m-2)}, U_{t}^{(m-2)}, U_{x}^{(m-2)}\right)\right) U^{(m-1)}\right\|_{\left(C^{0}[R(T)]\right)^{N}} \\
\leq & C_{20} C_{5}^{m-1}(G(\varepsilon)+\varepsilon)^{m}, \\
& \left\|\left(c^{2}\left(U^{(m-1)}, U_{t}^{(m-1)}, U_{x}^{(m-1)}\right)-c^{2}\left(U^{(m-2)}, U_{t}^{(m-2)}, U_{x}^{(m-2)}\right)\right) \frac{\partial^{2} U^{(m-1)}}{\partial x^{2}}\right\|_{\left(C^{0}[R(T)]\right)^{N}} \\
\leq & C_{21} C_{5}^{m-1}(G(\varepsilon)+\varepsilon)^{m}, \\
& \left\|\left(B\left(U^{(m-1)}\right)-B\left(U^{(m-2)}\right)\right) U^{(m-1)}\right\|_{\left(C^{0}[0, T]\right)^{N}} \leq C_{22} C_{5}^{m-1}(G(\varepsilon)+\varepsilon)^{m}, \\
& \left\|\left(\bar{B}\left(U^{(m-1)}\right)-\bar{B}\left(U^{(m-2)}\right)\right) U^{(m-1)}\right\|_{\left(C^{0}[0, T]\right)^{N}} \leq C_{23} C_{5}^{m-1}(G(\varepsilon)+\varepsilon)^{m},
\end{aligned}
$$

where $C_{20}, C_{21}, C_{22}$ and $C_{23}$ are positive constants depending only on $T$ and $T_{0}$.

Noting (4.40), by Lemma 3.1 there exists a positive constant $C_{24}=C_{24}\left(T, T_{0}\right)$ such that

$$
\left\|V^{(m)}\right\|_{\left(C^{1}[R(T)]\right)^{N}}=\left\|U^{(m)}-U^{(m-1)}\right\|_{\left(C^{1}[R(T)]\right)^{N}} \leq C_{24} C_{5}^{m-1}(G(\varepsilon)+\varepsilon)^{m} .
$$

Therefore, choosing $C_{5} \geq C_{24}$, we get (4.15). The proof of Theorem 1.1 is complete. 


\section{REMARKS}

Remark 5.1. Theorem 1.1 is still valid in the case of two-sided controls, provided that (1.14) is replaced by

$$
T>T_{0}>\frac{L}{c(0,0,0)},
$$

and there is an inhomogeneous control term $\bar{H}(t)$ on the right-hand side of (1.6).

Remark 5.2. Similar results hold for the following boundary conditions of coupled dissipative type:

$$
\begin{aligned}
& x=0: U_{x}-C(U) U_{t}=H(t), \\
& x=L: U_{x}+\bar{C}(U) U_{t}=0,
\end{aligned}
$$

where $C(U)=\left(c_{i j}(U)\right)_{N \times N}$ and $\bar{C}(U)=\left(\bar{c}_{i j}(U)\right)_{N \times N}$ are $C^{1}$ matrix functions with respect to their arguments. However, differently from boundary conditions (1.4) and (1.6), in the case of boundary conditions (5.2)-(5.3), in order to guarantee the well-posedness and the exact boundary null controllability for the forward mixed initial-boundary value problem, we should impose the following assumptions:

$$
\operatorname{det}\left(C(0)+\frac{1}{c(0,0,0)} I_{N}\right) \neq 0
$$

and

$$
\operatorname{det}\left(\bar{C}(0)+\frac{1}{c(0,0,0)} I_{N}\right) \neq 0
$$

for the boundary coupling matrices $C(U)$ and $\bar{C}(U)$. Then, combining the proof of Theorem 1.1 and the method presented in [3], one can realize the corresponding local exact boundary synchronization for the coupled system of wave equations (1.1) and (5.2)-(5.3) by $(N-1)$ boundary controls on $x=0$, provided that

$$
\sum_{j=1}^{N} c_{i j}(U) \stackrel{\text { def. }}{=} \widetilde{\widetilde{c}}(U)
$$

and

$$
\sum_{j=1}^{N} \bar{c}_{i j}(U) \stackrel{\text { def. }}{=} \widetilde{\bar{c}}(U),
$$

where $\widetilde{\widetilde{c}}(U)$ and $\widetilde{\widetilde{c}}(U)$ are independent of $i=1, \ldots, N$.

Remark 5.3. Theorem 1.1 and Remark 5.2 are also valid for the corresponding nonautonomous quasilinear system of wave equations, in which

$$
c=c\left(t, x, U, U_{t}, U_{x}\right), A=A\left(t, x, U, U_{t}, U_{x}\right)
$$

and

$$
B=B(t, U), \bar{B}=\bar{B}(t, U) \quad(\text { or } C=C(t, U), \bar{C}=\bar{C}(t, U))
$$

with suitable regularity with respect to $t$ and $x$. 
Remark 5.4. For the following more general coupled system of quasilinear wave equations:

$$
\frac{\partial^{2} U}{\partial t^{2}}-c^{2}\left(U, U_{t}, U_{x}\right) \frac{\partial^{2} U}{\partial x^{2}}+F\left(U, U_{t}, U_{x}\right)=0,
$$

where $F$ is a $C^{2}$ vector function with respect to its arguments and

$$
F(0,0,0)=0 .
$$

Thus, one can find $C^{1}$ matrices $A\left(U, U_{t}, U_{x}\right), \breve{A}\left(U, U_{t}, U_{x}\right)$ and $\hat{A}\left(U, U_{t}, U_{x}\right)$, such that

$$
F\left(U, U_{t}, U_{x}\right)=A\left(U, U_{t}, U_{x}\right) U+\breve{A}\left(U, U_{t}, U_{x}\right) U_{t}+\hat{A}\left(U, U_{t}, U_{x}\right) U_{x},
$$

and the corresponding local exact boundary synchronization can be realized, provided that $A, \breve{A}$ and $\hat{A}$ satisfy similar assumptions as in (1.11), respectively.

\section{Appendix. Proof of Lemma 4.2}

In order to get Lemma 4.2, it suffices to prove that for any given integer $m \geq 1$, there exists a unique $C^{2}$ solution $W^{(m)}=W^{(m)}(t, x)$ to the coupled system (4.23) of wave equations, which satisfies simultaneously the initial condition (4.28), the final condition (4.30) and the boundary condition (4.26) on $x=L$ as well as the estimates (4.31)-(4.33). To this end, we will use the constructive method suggested in [6, 8-10] with necessary modifications and improvements.

Let

$$
T^{(m)}=\int_{0}^{T_{0}} \inf _{0 \leq x \leq L} c\left(U^{(m-1)}(t, x), U_{t}^{(m-1)}(t, x), U_{x}^{(m-1)}(t, x)\right) \mathrm{d} t .
$$

Due to (1.14) and (4.19), for suitably small $\varepsilon>0$ we have

$$
T^{(m)}>2 L \text {. }
$$

Choose $T_{1}^{(m)}$ and $T_{2}^{(m)}$ such that

$$
\begin{aligned}
& \int_{0}^{T_{1}^{(m)}} \inf _{0 \leq x \leq L} c\left(U^{(m-1)}(t, x), U_{t}^{(m-1)}(t, x), U_{x}^{(m-1)}(t, x)\right) \mathrm{d} t \\
= & \int_{T_{2}^{(m)}}^{T_{0}} \inf _{0 \leq x \leq L} c\left(U^{(m-1)}(t, x), U_{t}^{(m-1)}(t, x), U_{x}^{(m-1)}(t, x)\right) \mathrm{d} t \\
= & L .
\end{aligned}
$$

By (1.14) and (4.19), for $\varepsilon>0$ small enough we have

$$
0<T_{1}^{(m)}<\frac{T_{0}-\delta}{2}<\frac{T_{0}}{2}<\frac{T_{0}+\delta}{2}<T_{2}^{(m)}<T_{0},
$$

in which

$$
\delta=\frac{1}{2}\left(T_{0}-2 \frac{L}{c(0,0,0)}\right) .
$$


(i) We first consider the forward mixed initial-boundary value problem for system (4.23) with the initial condition (4.28), the boundary condition (4.26) on $x=L$ and the following artificial Neumann boundary condition

$$
x=0: W_{x}^{(m)}=\mathfrak{F}(t), \quad 0 \leq t \leq \frac{T_{0}}{2},
$$

where $\mathfrak{F}(\cdot)$ is an arbitrarily given $C^{1}$ function of $t$, satisfying the following conditions of $C^{2}$ compatibility at the point $(t, x)=(0,0)$ :

$$
\left\{\begin{array}{l}
\mathfrak{F}(0)=\widetilde{\Phi}^{\prime}(0), \\
\mathfrak{F}^{\prime}(0)=\widetilde{\Psi}^{\prime}(0)
\end{array}\right.
$$

(see Rem. 1.5) and verifying

$$
\begin{aligned}
& \|\mathfrak{F}\|_{\left(C^{1}\left[0, \frac{T_{0}}{2}\right]\right)^{N-1}} \leq \varepsilon, \\
& \Omega(\eta \mid \dot{\mathfrak{F}}) \leq \Omega_{0}(\eta) .
\end{aligned}
$$

Due to (A.7)-(A.9), this artificial boundary condition (A.6) can be chosen to be independent of the iteration (i.e., independent of $m$ ), namely, we can take the same artificial boundary condition (A.6) in every iteration step (see also Rem. 1.6). Meanwhile, in order that the constants, obtained from all the estimates of the solutions in the iteration procedure, are also independent of $m$, each iteration should be discussed on the same domain, i.e., on

$$
R_{f}=\left\{(t, x) \mid 0 \leq t \leq \frac{T_{0}}{2}, 0 \leq x \leq L\right\}
$$

or

$$
R_{b}=\left\{(t, x) \mid \frac{T_{0}}{2} \leq t \leq T, 0 \leq x \leq L\right\}
$$

All these can be regarded as some modifications and improvements to the constructive method suggested in $[6,8-10]$.

Let

$$
\begin{gathered}
\widetilde{\Gamma}^{*}=\left\{\frac{\partial c}{\partial u_{k}}, \frac{\partial c}{\partial u_{k t}}, \frac{\partial c}{\partial u_{k x}}, \frac{\partial \widetilde{a}_{i j}}{\partial u_{k}}, \frac{\partial \widetilde{a}_{i j}}{\partial u_{k t}}, \frac{\partial \widetilde{a}_{i j}}{\partial u_{k x}}, \frac{\partial \widetilde{b}_{i j}}{\partial u_{k}}, \frac{\partial \widetilde{\bar{b}}_{i j}}{\partial u_{k}}\right\}, \\
i, j, k=1, \ldots, N .
\end{gathered}
$$

Obviously, there exists a positive constant $\widetilde{C}_{1}$ such that

$$
\omega\left(\eta \mid \widetilde{\Gamma}^{*}\right) \leq \widetilde{C}_{1} \omega\left(\eta \mid \Gamma^{*}\right) .
$$

Here and hereafter, for $i=1,2, \ldots, \widetilde{C}_{i}$ denote positive constants, $\widetilde{C}_{i}\left(T_{0}\right)$ denote positive constants depending only on $T_{0}$, and $\widetilde{C}_{i}\left(T, T_{0}\right)$ denote positive constants depending only on $T$ and $T_{0}$.

By Lemma 3.1, there exists a unique $C^{2}$ solution $W^{(m)}=W_{f}^{(m)}(t, x)$ on the domain $R_{f}$ (see (A.10)). In particular, noting (4.19), (4.21) and the properties of continuity modulus (see [12]), we have

$$
\begin{aligned}
& \left\|W_{f}^{(m)}(t, x)\right\|_{\left(C^{2}\left[R_{f}\right]\right)^{N-1}} \leq \widetilde{C}_{2}\left(T_{0}\right) \varepsilon \leq \widetilde{C}_{3}\left(T_{0}\right)(G(\varepsilon)+\varepsilon), \quad \forall(t, x) \in R_{f}, \\
& \omega\left(\eta \mid \frac{\partial^{2} W_{f}^{(m)}}{\partial t^{2}}\right)+\omega\left(\eta \mid \frac{\partial^{2} W_{f}^{(m)}}{\partial x^{2}}\right)+\omega\left(\eta \mid \frac{\partial^{2} W_{f}^{(m)}}{\partial t \partial x}\right) \\
& \leq \widetilde{C}_{4}\left(T_{0}\right)\left(\omega\left(\eta \mid \widetilde{\Phi}_{x x}\right)+\omega\left(\eta \mid \widetilde{\Psi}_{x}\right)+\Omega_{0}(\eta)+\varepsilon\left(\eta+\Omega_{1}(\eta)+(1+G(\varepsilon)+\varepsilon) \Omega\left(\eta \mid \widetilde{\Gamma}^{*}\right)\right)\right) \\
& \leq \widetilde{C}_{5}\left(T_{0}\right)\left(\omega\left(\eta \mid \Phi_{x x}\right)+\omega\left(\eta \mid \Psi_{x}\right)+\Omega_{0}(\eta)+(G(\varepsilon)+\varepsilon)\left(\eta+\Omega_{1}(\eta)+\Omega\left(\eta \mid \Gamma^{*}\right)\right)\right) .
\end{aligned}
$$


Thus we can uniquely determine the value of $\left(W_{f}^{(m)}, W_{f x}^{(m)}\right)$ on $x=L$ :

$$
x=L:\left(W_{f}^{(m)}, W_{f x}^{(m)}\right)=\left(a^{(m)}(t), \bar{a}^{(m)}(t)\right), \quad 0 \leq t \leq \frac{T_{0}}{2},
$$

and we have

$$
\begin{aligned}
& \left\|\left(a^{(m)}(t), \bar{a}^{(m)}(t)\right)\right\|_{\left(C^{2}\left[0, \frac{T_{0}}{2}\right]\right)^{N-1} \times\left(C^{1}\left[0, \frac{T_{0}}{2}\right]\right)^{N-1}} \leq \widetilde{C}_{6}\left(T_{0}\right)(G(\varepsilon)+\varepsilon), \\
& \omega\left(\eta \mid \ddot{a}^{(m)}\right)+\omega\left(\eta \mid \dot{\bar{a}}^{(m)}\right) \\
\leq & \widetilde{C}_{7}\left(T_{0}\right)\left(\omega\left(\eta \mid \Phi_{x x}\right)+\omega\left(\eta \mid \Psi_{x}\right)+\Omega_{0}(\eta)+(G(\varepsilon)+\varepsilon)\left(\eta+\Omega_{1}(\eta)+\Omega\left(\eta \mid \Gamma^{*}\right)\right)\right) .
\end{aligned}
$$

On the other hand, denoting

$$
Z_{f}^{(m)}=W_{f}^{(m)}-W_{f}^{(m-1)}, m=0,1,2, \ldots
$$

it is easy to see that $Z_{f}^{(m)}$ satisfies the following mixed initial-boundary value problem:

$$
\begin{aligned}
& \frac{\partial^{2} Z_{f}^{(m)}}{\partial t^{2}}-c^{2}\left(U^{(m-1)}, U_{t}^{(m-1)}, U_{x}^{(m-1)}\right) \frac{\partial^{2} Z_{f}^{(m)}}{\partial x^{2}}+\widetilde{A}\left(U^{(m-1)}, U_{t}^{(m-1)}, U_{x}^{(m-1)}\right) Z^{(m)} \\
& +\left(\widetilde{A}\left(U^{(m-1)}, U_{t}^{(m-1)}, U_{x}^{(m-1)}\right)-\widetilde{A}\left(U^{(m-2)}, U_{t}^{(m-2)}, U_{x}^{(m-2)}\right)\right) W_{f}^{(m-1)} \\
& -\left(c^{2}\left(U^{(m-2)}, U_{t}^{(m-2)}, U_{x}^{(m-2)}\right)-c^{2}\left(U^{(m-1)}, U_{t}^{(m-1)}, U_{x}^{(m-1)}\right)\right) \frac{\partial^{2} W_{f}^{(m-1)}}{\partial x^{2}}=0
\end{aligned}
$$

with the initial condition

$$
t=0:\left(Z_{f}^{(m)}, Z_{f t}^{(m)}\right)=(0,0), 0 \leq x \leq L,
$$

the boundary condition on $x=0$

$$
x=0: Z_{f x}^{(m)}=0
$$

and the boundary condition on $x=L$ :

$$
\begin{aligned}
& x=L: Z_{f}^{(m)}=0 \\
& x=L: Z_{f x}^{(m)}=0 \\
& x=L: Z_{f x}^{(m)}+\widetilde{\bar{B}}\left(U^{(m-1)}\right) Z_{f}^{(m)}=\left(\widetilde{\bar{B}}\left(U^{(m-2)}\right)-\widetilde{\bar{B}}\left(U^{(m-1)}\right)\right) W_{f}^{(m-1)} .
\end{aligned}
$$

Noting (4.19)-(4.20), (4.24) and (A.14), we get

$$
\begin{aligned}
I_{0} & \stackrel{\text { def }}{=} \\
& \leq\left(c^{2}\left(U^{(m-1)}, U_{t}^{(m-1)}, U_{x}^{(m-1)}\right)-c^{2}\left(U^{(m-2)}, U_{t}^{(m-2)}, U_{x}^{(m-2)}\right)\right) \frac{\partial^{2} W_{f}^{(m-1)}}{\partial x^{2}} \|_{\left(C^{0}\left[R_{f}\right]\right)^{N-1}} \\
I_{1} & \left.\stackrel{\text { def. }}{=} \|\left(\widetilde{A}\left(U^{(m-1)}, U_{t}^{(m-1)}, U_{x}^{(m-1)}\right)-\widetilde{A}\left(U^{(m-2)}, U_{t}^{(m-2)}, U_{x}^{(m-2)}\right)\right) W_{f}^{(m-1)}\right) \|_{\left(C^{0}\left[R_{f}\right]\right)^{N-1}} \\
& \leq \widetilde{C}_{9}\left(T_{0}\right) C_{5}^{m-1}(G(\varepsilon)+\varepsilon)^{m}, \\
I_{2} & \left.\stackrel{\text { def. }}{=} \|\left(\widetilde{\bar{B}}\left(U^{(m-1)}\right)-\widetilde{B}\left(U^{(m-2)}\right)\right) W_{f}^{(m-1)}\right) \|_{\left(C^{0}\left[R_{f}\right]\right)^{N-1}} \leq \widetilde{C}_{10}\left(T_{0}\right) C_{5}^{m-1}(G(\varepsilon)+\varepsilon)^{m} .
\end{aligned}
$$


Therefore, by Lemma 3.1, we have

$$
\left\|W^{(m)}-W^{(m-1)}\right\|_{\left(C^{1}\left[R_{f}\right]\right)^{N-1}} \leq \widetilde{C}_{11}\left(T_{0}\right)\left(I_{0}+I_{1}+I_{2}\right) \leq \widetilde{C}_{12}\left(T_{0}\right) C_{5}^{m-1}(G(\varepsilon)+\varepsilon)^{m} .
$$

In particular, we have

$$
\begin{aligned}
& \left\|\left(a^{(m)}(t)-a^{(m-1)}(t), \bar{a}^{(m)}(t)-\bar{a}^{(m-1)}(t)\right)\right\|_{\left(C^{1}\left[0, \frac{T_{0}}{2}\right]\right)^{N-1} \times\left(C^{0}\left[0, \frac{T_{0}}{2}\right]\right)^{N-1}} \\
\leq & \widetilde{C}_{13}\left(T_{0}\right) C_{5}^{m-1}(G(\varepsilon)+\varepsilon)^{m} .
\end{aligned}
$$

(ii) On the domain $R_{b}$ (see (A.11)), let $W_{b}^{(m)}(t, x) \equiv 0$. Obviously, $W=W_{b}^{(m)}(t, x)$ verifies system (4.23), final condition (4.30) and boundary conditions (4.26).

(iii) Thus, we can find the vector function $\left(c^{(m)}(t), \bar{c}^{(m)}(t)\right) \in\left(C^{2}[0, T]\right)^{N-1} \times\left(C^{1}[0, T]\right)^{N-1}$ (for example, by polynomial interpolation), such that

$$
\left(c^{(m)}(t), \bar{c}^{(m)}(t)\right)=\left\{\begin{array}{l}
\left(a^{(m)}(t), \bar{a}^{(m)}(t)\right), \quad 0 \leq t \leq \frac{T_{0}-\delta}{2}, \\
(0,0), \quad \frac{T_{0}+\delta}{2} \leq t \leq T
\end{array}\right.
$$

and $\left(c^{(m)}(t), \bar{c}^{(m)}(t)\right)$ verifies the boundary condition (4.26) at $x=L$ on the whole interval $0 \leq t \leq T$, moreover, we have

$$
\begin{aligned}
& \left\|\left(c^{(m)}(t), \bar{c}^{(m)}(t)\right)\right\|_{\left(C^{2}[0, T]\right)^{N-1} \times\left(C^{1}[0, T]\right)^{N-1}} \leq \widetilde{C}_{14}\left(T, T_{0}\right)(G(\varepsilon)+\varepsilon), \\
& \left\|\left(c^{(m)}(t)-c^{(m-1)}(t), \bar{c}^{(m)}(t)-\bar{c}^{(m-1)}(t)\right)\right\|_{\left(C^{1}[0, T]\right)^{N-1} \times\left(C^{0}[0, T]\right)^{N-1}} \\
\leq & \widetilde{C}_{15}\left(T, T_{0}\right) C_{5}^{m-1}(G(\varepsilon)+\varepsilon)^{m}, \\
& \omega\left(\eta \mid \ddot{c}^{(m)}\right)+\omega\left(\eta \mid \dot{\bar{c}}^{(m)}\right) \\
\leq & \widetilde{C}_{16}\left(T, T_{0}\right)\left(\omega\left(\eta \mid \Phi_{x x}\right)+\omega\left(\eta \mid \Psi_{x}\right)+\Omega_{0}(\eta)+(G(\varepsilon)+\varepsilon)\left(\eta+\Omega_{1}(\eta)+\Omega\left(\eta \mid \Gamma^{*}\right)\right)\right) .
\end{aligned}
$$

We now change the status of $t$ and $x$, and consider the following leftward mixed initial-boundary problem on the domain $R(T)=\{(t, x) \mid 0 \leq t \leq T, 0 \leq x \leq L\}$ for system (4.23) with the initial condition

$$
x=L: W_{l}^{(m)}=c^{(m)}(t), W_{l x}^{(m)}=\bar{c}^{(m)}(t), \quad 0 \leq t \leq T
$$

and the boundary conditions

$$
\begin{aligned}
& t=0: W_{l t}^{(m)}=\widetilde{\Psi}(x), \quad 0 \leq x \leq L, \\
& t=T: W_{l t}^{(m)}=0, \quad 0 \leq x \leq L
\end{aligned}
$$

where $\widetilde{\Psi}(x)$ is given by $(4.29)$.

Obviously, the corresponding conditions of $C^{2}$ compatibility are satisfied at the points $(t, x)=(0, L)$ and $(T, L)$, respectively. Hence, by Lemma 3.1, there exists a unique $C^{2}$ solution $W^{(m)}=W_{l}^{(m)}(t, x)$ on the domain $R(T)=\{(t, x) \mid 0 \leq t \leq T, 0 \leq x \leq L\}$, such that

$$
\begin{gathered}
\left\|W_{l}^{(m)}(t, x)\right\|_{\left(C^{2}[R(T)]\right)^{N-1}} \leq \widetilde{C}_{17}\left(T, T_{0}\right)(G(\varepsilon)+\varepsilon), \quad \forall(t, x) \in R(T), \\
\omega\left(\eta \mid \frac{\partial^{2} W_{l}^{(m)}}{\partial t^{2}}\right)+\omega\left(\eta \mid \frac{\partial^{2} W_{l}^{(m)}}{\partial x^{2}}\right)+\omega\left(\eta \mid \frac{\partial^{2} W_{l}^{(m)}}{\partial t \partial x}\right) \\
\leq \widetilde{C}_{18}\left(T, T_{0}\right)\left(\omega\left(\eta \mid \Phi_{x x}\right)+\omega\left(\eta \mid \Psi_{x}\right)+\Omega_{0}(\eta)+(G(\varepsilon)+\varepsilon)\left(\eta+\Omega_{1}(\eta)+\Omega\left(\eta \mid \Gamma^{*}\right)\right)\right) .
\end{gathered}
$$


Thus, $Z_{l}^{(m)}=W_{l}^{(m)}-W_{l}^{(m-1)}$ satisfies the mixed initial-boundary value problem of system (A.20) with the homogeneous boundary condition

$$
\begin{aligned}
& t=0: Z_{x}^{(m)}=0, \\
& t=T: Z_{x}^{(m)}=0
\end{aligned}
$$

and the initial condition

$$
x=L:\left(Z^{(m)}, Z_{x}^{(m)}\right)=\left(c^{(m)}(t)-c^{(m-1)}(t), \bar{c}^{(m)}(t)-\bar{c}^{(m-1)}(t)\right) .
$$

Combining (A.31), (A.36) and Lemma 3.1, we get

$$
\left\|W_{l}^{(m)}(t, x)-W_{l}^{(m-1)}(t, x)\right\|_{\left(C^{2}[R(T)]\right)^{N-1}} \leq \widetilde{C}_{19}\left(T, T_{0}\right) C_{5}^{m-1}(G(\varepsilon)+\varepsilon)^{m} .
$$

(iv) By the uniqueness of the $C^{2}$ solution for one-sided mixed initial-boundary value problem, based on the finite speed of wave propagation (see $[6,12,19,20]$ ), similarly to the argument on the exact boundary controllability in the case of linear wave equations (see [6,13]), we get that the $C^{2}$ function $W^{(m)}=W_{l}^{(m)}(t, x)$ on $R(T)=$ $\{(t, x) \mid 0 \leq t \leq T, 0 \leq x \leq L\}$ satisfies the system (4.23), the initial condition (4.29), the final condition (4.30) and the boundary condition (4.26) on $x=L$. Moreover, noting (A.36)-(A.37) and (A.41), we get (4.31)-(4.33). This concludes the proof of Lemma 4.3.

\section{REFERENCES}

[1] Hirokazu Fujisaka and Tomoji Yamada, Stability theory of synchronized motion in coupled-oscillator systems. Prog. Theor. Phys. 69 (1983) 32-47.

[2] Long $\mathrm{Hu}$, Fanqiong Ji and Ke Wang, Exact boundary controllability and exact boundary observability for a coupled system of quasilinear wave equations. Chin. Ann. Math. 34 (2013) 379-390.

[3] Long Hu, Tatsien Li, Bopeng Rao, Exact Boundary Synchronization for a Coupled System of 1-D Wave Equations with coupled boundary conditions of dissipative type. Commun. Pure Appl. Anal. 13 (2014) 881-901.

[4] Ch. Huygens, CEuvres Complètes, Vol. 15. Swets \& Zeitlinger B.V., Amsterdam (1967).

[5] Tatsien Li, Exact boundary controllability for quasilinear wave equations. J. Comput. Appl. Math. 190 (2006) 127-135.

[6] Tatsien Li, Controllability and Observability for Quasilinear Hyperbolic Systems. Vol. 3 of AIMS Series on Applied Mathematics. AIMS \& Higher Education Press (2010).

[7] Tatsien Li, Yi Jin, Semi-global $C^{1}$ solution to the mixed initial-boundary value problem for quasilinear hyperbolic systems. Chin. Ann. Math. 22 (2001) 325-336.

[8] Tatsien Li, Bopeng Rao, Local exact boundary controllability for a class of quasilinear hyperbolic systems. Chin. Ann. Math. 23 (2002) 209-218.

[9] Tatsien Li, Bopeng Rao, Exact boundary controllability for quasilinear hyperbolic systems. SIAM J. Control. Optim. 41 (2003) $1748-1755$.

[10] Tatsien Li, Bopeng Rao, Strong (weak) exact controllability and strong (weak) exact observability for quasilinear hyperbolic systems. Chin. Ann. Math. 31 (2010) 723-742.

[11] Tatsien Li, Bopeng Rao, Exact synchronization for a coupled system of wave equations with Dirichlet boundary controls. Chin. Ann. Math. 34 (2013) 139-160.

[12] Tatsien Li, Wenci Yu, Boundary Value Problems for Quasilinear Hyperbolic systems. Duke University Mathematics Series V (1985).

[13] Tatsien Li, Lixin Yu, Exact boundary controllability for 1-D quasilinear wave equations. SIAM J. Control. Optim. 45 (2006) $1074-1083$.

[14] Tatsien Li, Bopeng Rao, Long Hu, Exact boundary synchronization for a coupled system of 1-D wave equations. ESAIM: COCV 20 (2014) 339-361.

[15] J.-L. Lions, Contrôlabilité Exacte, Perturbations et Stabilization de Systèmes Distribués. Vol. I. Masson (1988).

[16] J.-L. Lions, Exact controllability, stabilization and perturbations for distributed systems. SIAM Rev. 30 (1988) 1-68.

[17] L.M. Pecora, T.L. Carroll, Synchronization in chaotic systems. Phys. Rev. Lett. 64 (1990) 821-824.

[18] S. Strogatz, SYNC: The Emerging Science of Spontaneous Order. THEIA, New York (2003).

[19] Zhiqiang Wang, Exact controllability for nonautonomous quasilinear hyperbolic systems. Chin. Ann. Math. 27 (2006) 643-656.

[20] Zhiqiang Wang, Exact boundary controllability for nonautonomous quasilinear wave equations. Math. Methods Appl. Sci. 30 (2007) 1311-1327.

[21] Chai Wah Wu, Synchronizaton in complex networks of nonlinear dynamical systems. World scientific (2007). 